Elie Azoulay

Giuseppe Citerio

Jean-François Timsit

\title{
The identity of Intensive Care Medicine
}

Published online: 15 January 2013

(C) Springer-Verlag Berlin Heidelberg and ESICM 2013

E. Azoulay ( $\bullet)$

AP-HP, Hôpital Saint-Louis, Medical ICU;

Université Paris-Diderot, Sorbonne Paris-Cité,

Faculté de médecine; INSERM, U717,

1 avenue Claude Vellefaux, 75010 Paris, France

e-mail: elieazoulay.icm@sls.aphp.fr

Tel.: +33142499421

Fax: +33 142499426

\section{G. Citerio}

Neuroanesthesia and Neurointensive Care Unit, Anestesia e Rianimazione, San Gerardo Hospital, via Pergolesi 33, 20900 Monza, Milan, Italy

\section{J.-F. Timsit}

Université Grenoble 1 (Joseph Fourrier) - Intensive Care Unit - Albert Michallon Teaching Hospital, U823,

27 Albert Bonniot Institute, 38076 La Tronche, France

Dear colleagues from all over the world,

It is a great privilege to become the new editors of Intensive Care Medicine (ICM). We feel honored to follow Massimo Antonelli, who brought the journal to its current position by means of scientific rigor and dedication. We are happy to serve a community of talented clinicians and scientists that includes specialists in all fields of medicine and high-level researchers committed to solve clinical problems through approaches ranging from basic science to patient studies. Our goal is to understand and to meet the specific needs of readers, authors, reviewers, editors, and all critical-care specialists around the world.

Gifted intensivists who are world-recognized experts in their fields have agreed to constitute the ICM board of editors. Equally crucial to quality is manuscript improvement and critical appraisal by reviewers. We are creating a board of reviewers and statisticians who will both select manuscripts and provide constructive comments to help authors improve their work. ICM readers and authors are privileged to be able to rely on a panel of highly committed reviewers who have spent many years offering their considerable scientific expertise at the service of the journal. We owe them a special debt of gratitude for all the hours of painstaking work performed anonymously and for no pay.

Even if many of you are not part of the current editorial board, you still have much to contribute to the journal: we are confident that all those who are keen to help ICM grow will be able to do so.

One of the first challenges for the new ICM team is to continue on its course and to change at the same time. ICM must continue to select the best clinical research works, based on fairness, objectivity, and an obsession with quality. Yet change is needed so that ICM can contribute to shape the continuously evolving world of healthcare science. New sections will be created and new communication media put to use to support our readers, authors, and reviewers.

From a practical standpoint, we will start with three changes in the ICM review process. First, Editorial Manager will check submissions for compliance with instructions to authors and Equator Network reporting guidelines. Manuscripts that do not comply will be sent back immediately for modification. Second, we will introduce an initial review step conducted by highly specialized editors, who will select manuscripts for examination by reviewers based on appropriateness for the ICM readership and on absence of serious flaws for which no solution is available. The result will be twofold: for a proportion of rejected manuscripts, the refusal decision will be communicated more quickly to the authors, who will then be able to seek a better home for their paper; and ICM reviewers will have fewer papers 
to review, which will translate in greater peace of mind for performing high-quality work on manuscripts that are amenable to improvement. Third, to improve the quality and speed of the peer review process, our board of highly committed and experienced reviewers will review large numbers of manuscripts. Statisticians, when needed, will also be involved in the editors' decisions. In addition, we will call on external reviewers, and all ICM authors will be deemed as potential ICM reviewers.

The new ICM sections include a "7-Day Profile Publication" section created to attract the best pieces of work by ensuring that the entire review process takes no longer than 7 working days. Moreover, in each issue of the journal, renowned specialists will update us with the latest "News" in their field, providing insights on the most relevant advances published in the medical literature, with special emphasis on those likely to influence clinical practice. Review articles will continue to be an important part of the journal. ICM needs state-of-the-art reviews that have attractive figures, clear tables, and conclusions of relevance to either research or clinical practice. We will also invite authors to contribute reviews in the format of "My Paper 20 Years Later". These reviews will provide a global picture of a given field while constituting an exercise in critical appraisal and providing an opportunity to synthesize the evidence, to define the up-to-date practices, and to suggest research ideas and future investigations. We will also invite specialists to contribute systematic reviews or meta-analyses. Another new section, "From the Inside," will contain poetry and personal experiences and opinions that you want to share with your colleagues. It will add depth to the journal, highlighting our specific focus on the sickest patients, most severely distressed families, and greatest personal challenges to nurses and other healthcare professionals. Finally, the new "Imaging in Intensive Care Medicine" section will attract self-explanatory images that are both original and didactic.

We live in an era of electronic social media. ICM is determined to use these new tools in a way that benefits the intensive-care community and patient care. Electronic social media can serve to disseminate important new information in a targeted manner that could help clinicians and researchers to find what they need within the now huge-and constantly growing - mass of published manuscripts. For each of their papers, authors will draft a 140-character tweet. Podcasts and videos including article summaries or interviews will be created by ICM for upload to social media sites and communication to the intensive-care community. Collecting your opinions on the journal, developing new ideas about the peer review process, critically appraising published articles, increasing the usefulness of ICM as an educational tool, and identifying new evidence that directly impacts patient care are among our major objectives. Finally, a graphic designer will create cartoons associated with selected articles and designed to stimulate creative scientific thinking, to illustrate the inevitable ambiguities that occur in science, and to emphasize crucial points.

Our view for your journal can be summarized in five points. First, we want ICM to be not only a source of excellent scientific information, but also a place where intensivists will find room to express the many other dimensions associated with the specific nature of their work with critically ill patients. Although poetry, cartoons, and social media may not increase the ICM impact factor, we believe these additions will enrich your work, both by better incorporating personal factors into your overall goal of providing your patients with the best possible care, and by helping you to rapidly identify which articles you need to read. Second, we want ICM to have your respect. We want you to select ICM as the preferred home for your article when you are still at the earliest stages of designing your research project. We are also determined to further expedite the peer review process. ICM is already among the journals with the shortest publication times, and we will keep speed among our key priorities. Third, ICM must continue to serve the entire critical-care community, not only in Europe but also throughout the world, by covering all fields of clinical critical-care research and all medical specialties. Fourth, although scientifically independent from the ESICM, the journal will continue to work very closely with the society. The two will evolve in the same directions, with the unswerving purpose of showcasing your excellent work and high level of creativity. Last, the journal needs to evolve in order to continue to lead in the ever-changing world of critical-care science. Our outcomes include your satisfaction; our ability to address your needs; and your willingness to select, download, read, and cite ICM articles. We want our new sections to catch and hold your interest. We want you to visit the ICM website frequently and to let us know what you think. If ICM helps you to continuously improve your clinical practice based on new scientific evidence; stimulates your desire to conduct clinical research; and evolves into a place where you feel at home, express yourself, and gain nourishment from the thoughts of others; then we will have reached our goal.

Dear readers, colleagues, friends, authors, reviewers, editors, please be ensured of our devotion to your journal, Intensive Care Medicine. 